\title{
Perfil epidemiológico e conhecimento populacional acerca do câncer de boca em uma campanha de prevenção e diagnóstico
}

\section{Epidemiological profile and population knowledge about oral cancer in a prevention and diagnosis campaign}

\begin{abstract}
Como citar este artigo: MORO, JULIANA S.; PALMA, VICTOR M., BARIN, LUISA

M.; FERRAZZO, KIVIA L.:

DÁNESI, CRISTIANE C.; Perfil epidemiológico e conhecimento populacional acerca do câncer de boca em uma campanha de prevenção e diagnóstico. Revista Saúde (Sta. Maria). 2019; 45 (3).
\end{abstract}

\section{Autor correspondente: \\ Nome: Juliana Silva Moro \\ E-mail: juliana.moroo@hotmail.com \\ Telefone: (55) 32209559 \\ Filiação Institucional: Universidade Federal de Santa Maria (UFSM), \\ Centro de Ciências da Saúde (CCS), Curso de Odontologia, Departamento de Patologia \\ Endereço para correspondência: \\ Rua: Av. Roraima no 1000 \\ Bairro: Camobi \\ Cidade: Santa Maria \\ Estado: Rio Grande do Sul \\ CEP: 97 105-900}

\section{Data de Submissão: \\ 24/01/2019}

Data de aceite:

19/12/2019

Conflito de Interesse: Não há conflito de interesse

\section{(cc) $\mathrm{BY}-\mathrm{NC}-\mathrm{ND}$}

\author{
Juliana Silva Moro, Victor Mello Palma, Luisa Machado Barin, Kivia Linhares Ferrazzo, \\ Cristiane Cademartori Danesi
}

\section{RESUMO}

Objetivo: Identificar o perfil epidemiológico dos participantes de uma campanha de prevenção e diagnóstico do câncer de boca "Maio Vermelho" e avaliar o conhecimento dos participantes sobre os fatores de risco dessa doença. Metodologia: Estudo transversal, obtido por meio dos dados contidos nas fichas da população participante da campanha, nos anos de 2015 e 2016, em uma cidade do interior do Rio Grande do Sul. Os participantes foram submetidos a um exame físico intra-bucal e receberam orientações sobre o câncer de boca. Foram coletados dados sociodemográficos e um questionário contendo perguntas sobre o câncer de boca foi aplicado. Para avaliar a associação entre as variáveis explicativas e o desfecho foi utilizado o teste de Regressão Logística, com resultados expressos em Odds Ratio, IC 95\%. Resultados: No total, 402 participantes foram incluídos na pesquisa. A maioria dos participantes eram mulheres (62\%), cor branca (85,9\%), faixa etária entre 30 e 59 anos (48,4\%), escolaridade maior que 8 anos (56\%), não tabagistas (77\%), não etilistas (77\%) e sem hábito de exposição solar (69\%). Em relação as perguntas sobre o câncer de boca, os participantes responderam que conheciam as causas da doença $(70 \%)$ e que o fumo (94\%), álcool $(76 \%)$ e a má higiene oral $(87 \%)$ eram considerados fatores de risco. Na análise multivariada, foi observado que as variáveis cor (OR=0.47; IC95\% 0.26-0.87), tabagismo (OR=0.54; IC95\% 0.33-0.89) e escolaridade (OR=0.58; IC95\% 0.37-0.90) influenciaram na resposta conhecer as causas do câncer bucal. Conclusão: Concluise que o perfil dos participantes da campanha não foi considerado o de maior risco para a doença e 0 nível de conhecimento sobre os fatores de risco foi considerado satisfatório.

PALAVRAS-CHAVE: Câncer de boca; Campanhas de saúde; Epidemiologia.

\section{ABSTRACT}

Objective: To identify the epidemiological profile of participants of a "Maio Vermelho" oral cancer prevention and diagnosis campaign and to assess participants' knowledge of risk factors for this disease. Methods: Cross-sectional study, obtained from the data contained in the records of the campaign's participating population, in 2015 and 2016, in a city in the interior of Rio Grande do Sul. Participants underwent an intra-oral physical examination and received guidance on oral cancer. Sociodemographic data were collected and a questionnaire containing questions about oral cancer was applied. To evaluate the association between the explanatory variables and the outcome, the Logistic Regression test was used, with results expressed as Odds Ratio, 95\% Cl. Results: In total, 402 participants were included in the study. Most participants were women $(62 \%)$, white $(85.9 \%)$, aged between 30 and 59 years (48.4\%), educational level higher than 8 years $(56 \%)$, non-smokers $(77 \%)$, non-alcoholics (77\%) and without habit. sun exposure (69\%). About the causes of oral cancer, participants answered that they knew the causes of oral cancer $(70 \%)$ and that smoking $(94 \%)$, alcohol $(76 \%)$ and poor oral hygiene (87\%) were risk factors. In the multivariate analysis, it was observed that the variables color $(\mathrm{OR}=0.47 ; 95 \% \mathrm{Cl} 0.26-0.87)$, smoking $(\mathrm{OR}=0.54 ; 95 \% \mathrm{Cl} 0.33-0.89)$ and education $(O R=0.58 ; 95 \% \mathrm{Cl} 0.37-0.90)$ influenced the response know the causes of oral cancer. Conclusion: It was concluded that the profile of the campaign participants was not considered to be the highest risk for the disease and the level of knowledge about the risk factors was considered satisfactory.

KEYWORDS: Mouth neoplasms; Health promotion; Epidemiology. 


\section{INTRODUÇÃO}

As neoplasias malignas da cavidade bucal, comumente conhecida como câncer de boca, apresentam características agressivas, como o potencial de invasão tecidual e metastático¹. O tipo histológico mais frequente é o carcinoma de células escamosa, visto que corresponde, aproximadamente, $90 \%$ de todas as malignidades orais ${ }^{2}$

Segundo o último relatório publicado pela Organização Mundial de Saúde, foram relatados 300 mil novos casos de câncer de boca em todo o mundo, sendo considerado um sério problema em saúde pública ${ }^{3}$. No Brasil, de acordo com o Instituto Nacional do Câncer (INCA), estima-se para 2018 que 11.200 novos casos de câncer de boca em homens e 3.500 em mulheres sejam diagnosticados no país4.

Em relação aos fatores de risco, a exposição ao tabaco, ao álcool e a radiação solar, assim como a higiene bucal precária e o vírus HPV, são considerados os principais para o desenvolvimento do câncer bucal2-5. Quando o tabagismo e etilismo estão associados, os riscos de desenvolvimento do câncer de boca se tornam potencialmente maiores ${ }^{6}$. Além disso, os casos de câncer de boca são frequentemente diagnosticados em homens, na faixa etária dos 50 a 59 anos ${ }^{7}$.

Apesar da sua fácil localização, o câncer de boca é frequentemente diagnosticado em fases avançadas ${ }^{2-4} \mathrm{e} \mathrm{em}$ indivíduos de baixa renda, com pouco acesso a serviços de saúde ${ }^{8}$. Ainda, o baixo nível de conhecimento dos indivíduos sobre os sinais e sintomas do câncer bucal, associados aos fatores de risco levam a um diagnóstico tardio ${ }^{9}$.

Nesse contexto, o conhecimento sobre o câncer de boca por parte da população constitui o primeiro passo para prevenção efetiva da doença, pois pacientes conscientes sobre os fatores de risco e a severidade da doença poderão procurar atendimento precocemente ${ }^{10}$. Assim, programas e campanhas divulgando informações e métodos de prevenções do câncer bucal são alternativas que podem ser realizadas em municípios e serviços de saúde, tendo em vista o baixo custo, a facilidade de deteç̧ão da lesão e os benefícios concedidos à população.

Diante do exposto, o objetivo deste trabalho foi identificar o perfil epidemiológico dos participantes de uma campanha de prevenção e diagnóstico do câncer de boca “Maio Vermelho". Além disso, buscou-se avaliar o conhecimento dos participantes sobre os fatores de risco da doença.

\section{MÉTODO}

Trata-se de um estudo transversal, obtido por meio dos dados contidos nas fichas da população participante da campanha de prevenção e diagnóstico precoce do câncer bucal "Maio Vermelho", nos anos de 2015 e 2016. Essa campanha estadual acontece anualmente desde 0 ano de 2011, na cidade de Santa Maria, interior do Rio Grande do Sul, sendo uma proposta idealizada pelo Estado do Rio Grande do Sul, Conselho Regional de Odontologia, Faculdades de Odontologia concomitantemente com as Secretarias Municipais de Saúde.

Os locais de coleta foram os postos de saúde e em uma praça localizada na região central da cidade com o auxílio de uma unidade móvel, cedida pela Prefeitura Municipal de Santa Maria. Primeiramente, os indivíduos que procuraram a campanha foram convidados a participar da pesquisa, sendo entregue um Termo de Consentimento Livre e Esclarecido (TCLE) e, em seguida, um questionário contendo as questões relacionadas ao câncer de boca foi aplicado. Posteriormente, o exame físico intra-bucal e as orientações quanto a deteç̧ão do câncer de boca foram realizados para todos os participantes que procuraram espontaneamente à campanha.

Os participantes diagnosticados com alguma lesão ou com necessidades de tratamentos foram encaminhados ao Departamento de Estomatologia do Curso de Odontologia da Universidade Federal de Santa Maria (UFSM).

Os critérios de inclusão foram: participantes que procuraram espontaneamente à campanha de prevenção e diagnóstico e que assinaram o TCLE. Os critérios de exclusão foram: participantes que se recusaram a responder ao questionário e que não assinaram o TCLE.

As variáveis coletadas das fichas dos pacientes foram: idade, sexo, cor, escolaridade, renda familiar e hábitos relacionados ao tabagismo, etilismo e exposição ao sol. Além disso, foram adquiridas informações sobre o conhecimento dos fatores de risco do câncer bucal, por meio de quatro perguntas contidas na ficha: (I) "Você conhece as causas do câncer de boca ?"; (II) "Fumar pode causar câncer de boca ?"; (III) "O álcool pode causar câncer de boca ?"; (IV) "Má higiene bucal pode causar câncer de boca?". As possíveis respostas para essas perguntas foram categorizadas como: sim, não e não soube responder.

A cor da pele foi dicotomizada em brancos e não brancos e o nível educacional foi dividido em: $>8$ anos e $\leq$ 8 anos de educação. A idade foi categorizada em 29 anos, 30-59 anos e +60 anos e a renda familiar foi dicotomizada em $>1$ salário e $\leq 1$ salário. Posteriormente a coleta dos dados, esses foram digitalizados em uma planilha eletrônica e analisados descritivamente. Após a análise descritiva, foi realizado o teste de regressão Logística para avaliar a associação entre o desfecho, retirado da pergunta "Você conhece as causas do câncer de boca?", e as variáveis explicativas. As variáveis explicativas que apresentaram valor de $p \leq 2,0$ na análise não ajustada foram incluídas na regressão. Na análise multivariada, foi utilizado o processo Backward Stepwise. Valores de $p \leq 0,05$ foram considerados estatisticamente significativos. Os resultados foram descritos em Odds Ratio (OR) com IC 95\%. O programa utilizado para a análise estatística foi o Stata 13.0 (Stata Corporation; College Station, TX, USA).

O estudo foi aprovado pelo Comitê de Ética em Pesquisa com Seres Humanos da UFSM (CAAE nº $66228617.3 .0000 .5346)$.

\section{RESULTADO}

Dentre as 532 fichas analisadas, 402 foram incluídas no estudo por apresentarem dados completos. A tabela 1 
apresenta a análise descritiva do perfil dos participantes. De acordo com os resultados, a maioria dos participantes eram do sexo feminino (62\%), faixa etária entre 30 a 59 anos $(48,4 \%)$ e cor da pele branca $(85,9 \%)$. Em relação a escolaridade e a renda familiar dos indivíduos, a maior parte apresentava mais de 8 anos de estudo (56\%) e menor ou igual a um salário mínimo (51\%). Além disso, observou-se que a maioria dos participantes eram não tabagistas (77\%), não etilistas (77\%) e não costumavam ficar expostos ao sol (69\%).

Tabela 1. Perfil dos participantes da campanha Maio Vermelho, Rio Grande do Sul, 2015 e 2016 ( $n=402)$.

\begin{tabular}{|c|c|}
\hline Variáveis & n (\%) \\
\hline \multicolumn{2}{|l|}{ Gênero } \\
\hline Feminino & $249(62)$ \\
\hline Masculino & $153(28)$ \\
\hline \multicolumn{2}{|l|}{ Idade } \\
\hline$\leq 29$ anos & $17(4,4)$ \\
\hline 30-59 anos & $193(48,4)$ \\
\hline+60 anos & $192(47,2)$ \\
\hline
\end{tabular}

\section{Cor da pele}

$\begin{array}{ll}\text { Branca } & 349(85,9) \\ \text { Não-Branca } & 53(14,1)\end{array}$

\section{Escolaridade}

$\begin{array}{ll}>8 \text { anos } & 224(56) \\ \leq 8 \text { anos } & 178(44)\end{array}$

Renda familiar

$\begin{array}{ll}>1 \text { salário } & 196(49) \\ \leq 1 \text { salário } & 206(51)\end{array}$

Tabagismo

$\begin{array}{ll}\text { Não } & 301(77) \\ \text { Sim } & 91(23)\end{array}$

Etilismo

Não $\quad 302(77)$

Sim $100(23)$

Exposição ao Sol

\begin{tabular}{lll}
\hline Não & $276(69)$ \\
\multirow{2}{*}{$\operatorname{Sim}$} & $126(31)$ \\
\hline & TOTAL & $402(100)$ \\
\hline
\end{tabular}

Fonte: Arquivo próprio.

Referente as perguntas sobre os fatores de risco, a maioria dos participantes responderam que conheciam as causas do câncer de boca (70\%) e que o fumo (94\%), álcool (75\%) e a má higiene oral (87\%) podem estar associados a ocorrência do carcinoma (Tabela 2)

Tabela 2. Frequência das respostas do questionário aplicado, Rio Grande do Sul, 2015-2016 (n=402).

\begin{tabular}{ll}
\hline Perguntas & $\mathrm{n}(\%)$ \\
\hline Você sabe a causa do câncer? & $283(70)$ \\
Sim & $119(30)$ \\
Não & - \\
Não soube responder & \\
Fumar pode causar câncer de boca? & $377(94)$ \\
Sim & $21(5)$ \\
Não & $4(1)$ \\
Não soube responder & \\
Oálcool pode causar câncer de boca? & $302(75)$ \\
Sim & $86(21)$ \\
Não & $14(4)$ \\
Não soube responder & \\
Má higiene bucal pode causar câncer de boca? & $350(87)$ \\
Sim & $41(10)$ \\
Não & $11(3)$ \\
Não soube responder & $402(100)$ \\
\hline TOTAL &
\end{tabular}

Fonte: Arquivo próprio. 
Na análise bivariada, verificou-se que as variáveis cor, escolaridade, etilismo e tabagismo apresentaram associação ao desfecho. Entretanto, após a análise ajustada, a variável etilismo perdeu significância estatística permanecendo as variáveis cor da pele, escolaridade e tabagismo associados a conhecer as causas do câncer de boca $(p \leq 0,05)$.

De acordo com os resultados ilustrados na tabela 3, observou-se que indivíduos cuja cor da pele era não branca apresentaram 53\% (IC95\%; 0,26-0,87, p<0,016) menos chances de ter conhecimento sobre as causas do câncer de boca quando comparados aos indivíduos com cor da pele branca.

No que se refere a escolaridade, as chances de conhecer sobre as causas do câncer de boca foi 42\% (IC95\%0,37$0,90, p<0,016$ ) menor em indivíduos com escolaridade $\leq 8$ anos em relação aos com escolaridade $>8$ anos.

Em relação ao tabagismo, os fumantes apresentaram $46 \%$ (IC95\% 0,33-0,89, p<0,017) menos chances de conhecer as causas do câncer de boca em relação aos não-fumantes.

Tabela 3. Associação entre o perfil dos pacientes da campanha Maio Vermelho e o desfecho conhecer as causas do câncer de boca, Rio Grande do Sul, 2015-2016 (n=402).

\begin{tabular}{|c|c|c|c|c|c|}
\hline Variáveis & $n(\%)$ & $\begin{array}{l}\text { OR } \\
\text { Bivariada } \\
\text { (IC 95\%) }\end{array}$ & $p$ & $\begin{array}{l}\text { OR } \\
\text { Ajustada } \\
\text { IC (95\%) }\end{array}$ & $p$ \\
\hline \multicolumn{6}{|l|}{ Gênero } \\
\hline Feminino & $175(70)$ & 1 & & & \\
\hline Masculino & $108(70)$ & $1,01(0,65-1,57)$ & 0,948 & - & - \\
\hline \multicolumn{6}{|l|}{ Idade } \\
\hline$\geq 29$ anos & $14(82)$ & 1 & & & \\
\hline $30-59$ anos & $141(73)$ & $0,58(0,16-2,10)$ & 0,408 & & \\
\hline+60 anos & $128(67)$ & $0,42(0,11-1,54)$ & 0,195 & - & - \\
\hline \multicolumn{6}{|l|}{ Cor da pele } \\
\hline Branca & $253(72)$ & 1 & & 1 & \\
\hline Não-Branca & $30(57)$ & $0,49(0,27-0,89)$ & 0,020 & $0,47(0,26-0,87)$ & 0,016 \\
\hline \multicolumn{6}{|l|}{ Escolaridade } \\
\hline$>8$ anos & $168(75)$ & 1 & & 1 & \\
\hline$\leq$ 8anos & $115(64)$ & $0,60(0,39-0,93)$ & 0,024 & $0,58(0,37-0,90)$ & 0,016 \\
\hline
\end{tabular}

\section{DISCUSSÃO}

Neste estudo, avaliamos o perfil epidemiológico e o conhecimento dos pacientes sobre os fatores de risco do câncer bucal em uma campanha de prevenção realizada em uma cidade no interior do Brasil. Os resultados demonstraram que a população aderente as campanhas não se enquadram em um perfil de risco para o câncer bucal. Além disso, 0 conhecimento dos participantes sobre os fatores de risco foi satisfatório, sendo esse influenciado pelas variáveis cor da pele, nível de escolaridade e tabagismo.

Em relação ao perfil dos indivíduos entrevistados durante a campanha, a maioria eram mulheres, cor da pele branca e idade entre 30 a 59 anos. Geralmente, mulheres procuram mais os serviços de saúde do que os homens ${ }^{11}$, podendo ser esse o motivo relacionado a maior participação feminina na presente campanha. Tais dados vão ao encontro dos achados de Nemoto et al..$^{12}$ e Quitino et al. ${ }^{13}$, nos quais foram relatados maiores participantes mulheres, com idade menor do que 50 anos, durante campanhas de prevenção ao câncer de boca.

No que se refere aos hábitos, como o uso de tabaco, álcool e exposição solar, a maioria não era adepta, tal qual no estudo de Nemoto et al. ${ }^{12}$ e Ravazi et al. ${ }^{14}$ Tendo em vista que esse perfil não é considerado de maior risco ${ }^{7}$, salienta-se a necessidade de delinear estratégias para conscientizar indivíduos de maior risco sobre a importância do câncer bucal. Nesse contexto, Ismail et al15 elaboraram estratégias para chamar a atenção dos grupos expostos ao desenvolvimento do câncer bucal, no estado de Michigan, Estados Unidos da América. Os autores realizam campanhas 
em massa, utilizando diferentes meios de comunicação (anúncios em jornais, rádios e outdoors), com mensagens direcionadas aos grupos de risco sobre a importância da detecção precoce do câncer de boca, durante dois anos. Segundo o estudo, os pesquisadores observaram que as campanhas direcionadas e realizadas em diferentes mídias, aumentaram a conscientização sobre o câncer de boca e a procura por profissionais da saúde, principalmente cirurgiões dentistas, para realizar exames de detecção de lesões.

De acordo com os resultados encontrados, a maioria dos participantes apresentam conhecimento sobre fatores de risco do câncer bucal. Na pesquisa de Shimpi et al..$^{16}$, os resultados para o tabaco foram semelhantes aos encontrados nesse estudo (94\%), porém menores para o álcool (40\%). A higiene oral também foi relatada como um preditor, corroborando com o de Villa et al. ${ }^{17}$. Entretanto, estes resultados devem ser avaliados com cautela, pois a maioria dos participantes da campanha não são populações expostas aos riscos para o carcinoma oral ${ }^{12}$.

No contexto do nível educacional, observou-se nesse estudo que indivíduos com menor escolaridade conheciam menos as causas do câncer bucal em relação aqueles com maior escolaridade. Esse resultado está de acordo com o estudo de Al-Mawari et al. ${ }^{18} \mathrm{em}$ que se estudou o grau de conhecimento do câncer bucal na população do lêmen. Os autores observaram que os indivíduos com maior nível educacional apresentaram maiores conhecimentos sobre as causas do câncer de boca comparados aos com baixo nível educacional, demonstrando que conhecimento sobre essa neoplasia maligna pode estar diretamente relacionada à escolaridade do indivíduo.

Na presente pesquisa, participantes não brancos apresentaram menores chances de conhecer as causas do câncer de boca. Tais resultados corroboram com o estudo de Osazuwa-Peters et al..$^{19} \mathrm{em}$ que ao comparar as respostas dos indivíduos afro-americanos em relação aos caucasianos sobre os fatores de risco da doença, os autores observaram que os afro-americanos apresentaram menor probabilidade de apresentar alto conhecimento sobre o câncer bucal. Segundo o estudo, esse achado alerta sobre as desigualdades raciais em relação ao conhecimento das causas do câncer de boca e, principalmente, aos piores índices de sobrevida observados nos pacientes não brancos estarem atribuídos a procura tardia para tratamentos.

Além disso, outro achado importante nesse estudo se refere aos indivíduos fumantes conhecer menos as causas do câncer bucal. Esses pacientes são mais susceptíveis a desenvolver a doença ${ }^{3}$, necessitando maior atenção por parte dos profissionais de saúde, a fim de informa-los a respeito dessa neoplasia maligna e incentiva-los a mudança de comportamento. Corroborando com nossos achados, o estudo de Khwankong et al. ${ }^{20}$ também observou que os pacientes fumantes não tinham conhecimento sobre os sinais e fatores predisponentes ao câncer bucal.

Limitações pertinentes à utilização de fichas clínicas devem ser consideradas nesse estudo, pois existem informações incompletas contidas que não puderam ser avaliadas, diminuindo o número da amostra. Entretanto, apesar dessa limitação, pode-se observar nesse estudo, informações importantes em relação ao perfil dos participantes das 8 Saúde (Santa Maria), Vol. 45, n. 3, p. 1-11, set./dez., 2019 campanhas de prevenção e o conhecimento a respeito dos fatores de risco da doença. Esses dados podem auxiliar futuramente, no planejamento de campanhas de prevenção e diagnóstico do câncer de boca.

\section{CONSIDERAÇÕES FINAIS}

Com base nos resultados, conclui-se que o perfil dos participantes que procuraram espontaneamente a campanha de prevenção e diagnóstico precoce do câncer de boca não foram considerados o principal grupo de risco da doença. Esse resultado alerta sobre a baixa efetividade das campanhas de prevenção e diagnóstico e a necessidade de reformular as estratégias para atingir os grupos de maior risco para o câncer de boca, como homens de idade avançada tabagistas e etilistas

Além disso, os níveis de conhecimento dos participantes sobre os fatores de risco foram considerados satisfatórios, sendo que as características cor da pele, escolaridade e tabagismo podem influenciar sobre esse conhecimento.

\section{REFERÊNCIAS}

1. Coleman WB, Rubinas TC. Neoplasia. Molecular Pathology (Second Edition). 2018

2. Kumar M, Nanavati R, Modi TG, et al. Oral cancer: etiology and risk factors: A review. J Cancer Res Ther 2016;12:458-63.

3. BW Stewart, CP Wild. World Cancer Report. IARC Press. 2014

4. Brasil. Ministério da Saúde. Instituto Nacional do Câncer. Estimativa 2018 - Incidência de câncer no Brasil. 2018

5. Speight PM, Farthing PM. The pathology of oral cancer. Br. Dent. J. 2018;225: 841-7.

6. Morse DE, Walter JP, Cleveland D, et al. Smoking and drinking in relation to oral cancer and oral epithelial dysplasia. Cancer Causes Control. 2007;18: 919-29.

7. Moro JS, Cunha MM, Ardenghi TM, et al. Câncer de boca e orofaringe: epidemiologia e análise da sobrevida. ISSN 2236-5834 
Einstein (São Paulo). 2018; 16(2):1-5

8. Borges DML, Sena MF, Ferreira MA, et al. Mortality for oral cancer and socioeconomic status in Brazil. Cad. Saúde Pública. 2009:25:321-7.

9. Baumman $\mathrm{E}$, Koller $\mathrm{H}$, Wiltfang $\mathrm{J}$, et al. Challenges of early detection of oral cancer: raising awareness as a first step to successful campaigning. Health Educ Res. 2016;3:136-45

10. Oliveira LR, Ribeiro-Silva A, Zucoloto S. Perfil da incidência e da sobrevida de pacientes com carcinoma epidermóide oral em uma população brasileira. J Bras Patol Med Lab. 2006;42:385-92.

11. Figueiredo W. Assistência à saúde dos homens: um desafio para os serviços de atenção primária. Ciênc Saúde Coletiva 2005; 10:105-9

12. Nemoto RP, Victorino AA, Pessoa GB, et al. Oral cancer preventive campaigns: are we reaching the real target? Braz J Otorhinolaryngol. 2015;81:44-9..

13. Quirino MRS, Gomes FC, Marcondes MS, et al. Avaliação do conhecimento sobre o câncer de boca entre participantes de campanha para prevenção e diagnóstico precoce da doença em Taubaté - SP. Rev. Odontol. UNESP. 2006; 35(4)

14. Ravazi SM, Tahani B, Nouri S, et al. Oral cancer knowledge and practice among dental patients and their attitude towards tobacco cessation in Iran. Asian Pac J Cancer Prev. 2015;16:5439-44.

15. Ismail Al, Jedele JM, Lim S, et al. Marketing campaign to promote screening for oral câncer. J Am Dent Assoc 2012; 143:57-66.

16. Shimpi N, Jethwani M, Bharatkumar A, et al. Patient awareness/knowledge towards oralcancer: a cross-sectional survey. BMC Oral Health. 2018;18:86.

17. Villa A, Kreimer AR, Pasi M, et al. oral cancer knowledge: a survey administered to patients in dental departments at large Italian Hospitals. J Cancer Educ. 2011;26:505-9.

Saúde (Santa Maria), Vol. 45, n. 3, p. 1-11, set./dez., 2019
18. Al-Mawari SA, Addas A, Tarakji B, et al. Public awareness and knowledge of oral cancer in yemen. Asian Pac J Cancer Prev. 2015:15:10861-5.

19. Osazuwa-Peters N, Boakye EA, Hussaini AS, et al. Characteristics and predictors of oral cancer knowledge in a predominantly African American Community. PLoS One. 2017; 12.

20. Khwankong S, Sriplung H, Kerdpon D. Knowledge and health belief attitudes of oral cancer and itsscreening among at-risk Southern Thai Muslims. J Canc Educ. 2016;33:615-21. 